\title{
Improving Cancer Care
}

\section{Quality Oncology Practice Initiative Turns Seven}

\author{
By Joseph O. Jacobson, $M D$
}

\begin{abstract}
North Shore Medical Center, Salem, MA; and Quality Oncology Practice Initiative Steering Committee, American Society of Clinical Oncology, Alexandria, VA
\end{abstract}

It is fitting that the current issue of Journal of Oncology Practice highlights the ASCO Quality Oncology Practice Initiative (QOPI). Seven years ago, Joseph Simone, MD, then chair of the National Cancer Policy Board of the Institute of Medicine, submitted a modest proposal to ASCO. He suggested the funding of a feasibility study to determine if a small cadre of highly selected oncologists would be willing to voluntarily measure the quality of care they provide and share those results with one another and with their colleagues. The details of Simone's vision and the remarkable history of QOPI are summarized in his commentary. Simone's vision for a quality measurement system has been realized far beyond his initial expectations. QOPI has grown to include more than 500 practices. With the support of ASCO, QOPI remains true to Simone's vision as a voluntary, oncologist-led measurement system designed to encourage practices to regularly monitor and improve the care they provide.

Just as a stethoscope helps a physician make a diagnosis, QOPI is a tool that allows practices to identify opportunities to provide better care. QOPI measures processes of care routinely provided in outpatient oncology practices. Measurement alone, however, does not translate into improvement. Although highlighting a flawed process may lead to temporary improvement, benefits are usually short lived. Sustained improvement in a process requires the application of the principles and tools of quality improvement, and the identification and sharing of best practices are often beneficial.

Siegel et al define the next step toward Simone's goal of improving adult oncology care. The National Cancer Institute Community Cancer Centers Program (NCCCP) was established as a consortium of independent oncology practices located throughout the country that agreed to work collaboratively to improve performance across the cancer care continuum. The NCCCP's Quality of Care Subcommittee, led by Siegel and working in close collaboration with ASCO, has used QOPI to measure practice performance among 11 NCCCP practices. The collaboration enables centers excelling at particular measures to share their best practices with others, and participants are encouraged to incorporate the new processes into their workflow. The details of the program, along with representative performance data, are included in their article.

Blayney et al highlight a successful collaboration between Blue Cross Blue Shield of Michigan (BCBSM) and practicing medical oncologists. Recognizing that some Michigan practices might not be able to participate in QOPI, either because of limited staff or funds to undertake data collection, BCBSM created a program to provide funding for QOPI participation. This approach led to a fourfold increase in participation by medical oncologists in Michigan. In addition, BCBSM has facilitated the creation of the Michigan Oncology Quality Consortium, which, like the NCCCP, will enable oncologists to share best practices.

McNiff et al describe another step in the evolution of QOPI. On the basis of feedback from long-term QOPI participants and mounting pressure from payers, ASCO has created the QOPI Certification Program. This program will allow QOPI practices to take the next step in practice improvement. A certified practice will need to achieve a minimum threshold performance on a selected set of QOPI performance indicators and demonstrate that a set of chemotherapy safety standards are in place in the practice. Like the existing QOPI program (which will remain in place), participation in the QOPI Certification Program is voluntary.

Dodgion and Greenberg provide an important update on the use of population-based registries to address variation in cancer care. The authors suggest that the rapid adoption of electronic health records in oncology will soon become a source of highly granular and timely clinical information that will lead to the development valuable clinical registries to focus improvement efforts. ASCO has developed the Breast Cancer Registry Pilot Program, which uses a Web-enabled application based on the ASCO Breast Cancer Treatment Plan and Summary template. This methodology may eventually replace the current approach to QOPI data collection, which requires manual retrospective medical record abstraction and data entry.

The QOPI program is being increasingly recognized as an important example of a medical subspecialty society that has responded quickly and successfully to the national need to address quality of care. Schneider, a senior scientist at the RAND Corporation and co-investigator of the earlier ASCOsponsored National Initiative on Cancer Care Quality, provides the final commentary in this issue, framing the QOPI program in the context of other national physician organization-sponsored quality initiatives.

Joseph O. Jacobson, MD, is chair of the Department of Medicine and an oncology specialist at North Shore Medical Center in Salem, MA, and chair of the ASCO Quality Oncology Practice Initiative Steering Committee. Contact him at jjacobson@partners.org.

DOI: 10.1200/JOP.091055 